NOVA

University of Newcastle Research Online

nova.newcastle.edu.au

Kouretzis, George P.; Sheng, Daichao; Wang, Dong "Numerical simulation of cone penetration testing using a new critical state constitutive model for sand" Computers and Geotechnics Vol. 56, p. 50-60 (2014)

Available from: http://dx.doi.org/10.1016/j.compgeo.2013.11.002

Accessed from: $\underline{\text { http://hdl.handle.net/1959.13/1056705 }}$ 


\title{
NUMERICAL SIMULATION OF CONE PENETRATION TESTING USING A NEW CRITICAL STATE CONSTITUTIVE MODEL FOR SAND
}

\author{
George P. Kouretzis ${ }^{* 1}$, Daichao Sheng ${ }^{1}$, and Dong Wang ${ }^{2}$ \\ ${ }^{1}$ ARC Centre of Excellence for Geotechnical Science and Engineering, Faculty of Engineering and Built \\ Environment, The University of Newcastle, Australia \\ ${ }^{2}$ Centre for Offshore Foundation Systems, The University of Western Australia
}

\begin{abstract}
A new perspective on the numerical simulation of cone penetration in sand is presented, based on an enhanced critical state model implemented in an explicitintegration finite element code. Its main advantage, compared to similar studies employing simpler soil models, is that sand compressibility can be described with a single set of model parameters, irrespective of the stress level and the sand relative density. Calibration is based on back-analysis of published centrifuge experiments, while results of the methodology are also compared against independent tests. Additional analyses are performed to investigate sand state effects on cone penetration resistance, in comparison with empirical expressions from the literature.
\end{abstract}

Keywords: cone penetration; sand; critical state model; relative density; centrifuge tests.

\section{INTRODUCTION}

A number of studies have been published in the modern literature on the numerical simulation of moving boundary problems in cohesionless soils [e.g. 1, 2, 3, 4, 5]. A common assumption among these studies is the simulation of the nonlinear response of sand with simple constitutive models, such as the Drucker-Prager model. However, this approach has some limitations with regard to the numerical simulation of the cone resistance developing during the cone penetration test (CPT), which depends chiefly on the stress level, the sand relative density, and the sand compressibility $[6,7]$. The latter two parameters related to sand response cannot be directly quantified with simple models such as the Drucker-Prager and the MohrCoulomb models. On the other hand, the use of a very complex constitutive model to realistically simulate cone penetration in sand can be extremely challenging, keeping in mind that the problem involves high mesh distortion and frictional contact in finite element analysis.

Aiming at a more robust simulation of large deformation problems in sand, by straightforwardly accounting for the key parameters affecting resistance development, a new constitutive model is developed here. The model balances between flexibility, simplicity and computational efficiency, with particular emphasis on the latter two aspects. The constitutive model is expected to have about the same level of flexibility in capturing different soil behaviors as existing cone-cap models [e.g. 8, 9, 10, 11, 12, 13] or bounding surface models [e.g. 14, 15, 16, 17, 18, 19, 20], but with fewer material parameters and a better mathematical smoothness, in order to render it ideal for the simulation of large-strain problems. Furthermore, its material parameters can be easily determined from conventional laboratory tests, such as

\footnotetext{
* Corresponding author - email: Georgios.Kouretzis@newcastle.edu.au tel. +61 24921 6449, postal address: EA Building, University of Newcastle, Callaghan NSW 2308, Australia
} 
triaxial tests. The typical features of soil behavior considered here include those observed for sands with different densities, but also clays with different overconsolidation ratios. In this context, we exclude complicating features in soil behavior such as partial saturation, initial structure, anisotropy, rate dependency and hysteresis under cyclic loading. While these features are unquestionably very important, they are not involved in quasi-static moving boundary problems as the one we focus herein, and they can be considered separately without causing major changes to the model framework.

To simulate the boundary value problem, we implemented the constitutive model developed in the explicit module of the commercial finite element code ABAQUS [21], as described in the following paragraphs. Calibration of the constitutive model and verification of the results of the developed numerical methodology in based on centrifuge test results in Fontainebleau sand of varying density, from five independent laboratories [7, 22].

A series of parametric analyses regarding sand state effects on the cone resistance is accordingly presented, and the results are compared against well-established empirical relations proposed for the estimation of the sand relative density, $D_{r}$ and the state parameter $\psi$ [23] from CPT measurements. The comparison suggests that the proposed methodology is able to capture the effects of sand density on large displacement problems, if properly calibrated to account for sand-specific parameters. Although the centrifuge experiments, as well as the numerical analyses, refer to dry sand only, the effect of the degree of saturation on the developing cone resistance has been found to be trivial $[24,25,26]$, thus it can be argued that the outcome of this work applies to saturated sands too.

Apart from the above, a relatively detailed description of the numerical methodology is presented, as it could be of interest for researchers and practicing engineers working in the field of simulation of (very) large displacement problems in cohesionless soils.

\section{THE CONSTITUTIVE MODEL}

The sand model is based on the versatile model of Yao et al. [27] which in turn has roots in the popular Modified Cam Clay (MCC) model. The model of Yao et al. [27] uses two state parameters similar but not identical to the state parameter concept of Been and Jefferies [23] to capture the behavior of sands and overconsolidated clays, and it reduces to its base MCC model for normally consolidated clays. It also employs a complex hardening law to capture the density-dependent contraction/dilation behavior of normally- and overconsolidated sands. In this paper, the model of Yao et al. [27] is further simplified in terms of the hardening law, the compressibility and the three-dimensional generalisation, without losing its versatility. The enhanced model has only 7 material parameters that are all associated with a clear (at least phenomenological) physical meaning, and thus can be directly determined from conventional laboratory tests.

\subsection{Density-dependent peak shear strength and compressibility}

One of main features of the model is that the peak shear strength and the compressibility of a soil depend on its initial void ratio $e_{0}$. To characterize this dependency, we first define a number of straight lines in the $v$-Inp space, where $v$ is the specific volume $v=1+e$ and $p$ is the mean effective stress (Figure 1). As in all critical state models, we assume there is a straight line representing the ultimate state where the volume of the soil remains constant with increasing shear strain. This line is the so-called critical state line (CSL) and its slope is assumed to be $\lambda_{c}$. We also assume that the unloading line (with decreasing mean effective stress) has a 
slope $\kappa$, irrespective of the initial void ratio or the initial stress. Another straight line representing the isotropic compression of the densest possible state of the soil is referred to as the densest compression line (DCL) and is assumed to have the same slope as the unloading line. We further define a reference compression line (RCL) that lies above the CSL, separated by a vertical distance of $\left(\lambda_{c}-K\right) \ln 2$. This line is recognized as the normal consolidation line (NCL) in the MCC model, and is not the locus of points of maximum void ratio. We will demonstrate later that a soil with an initial void ratio on or above the RCL will exhibit only volume contraction, but below the RCL, both volume contraction and dilation.

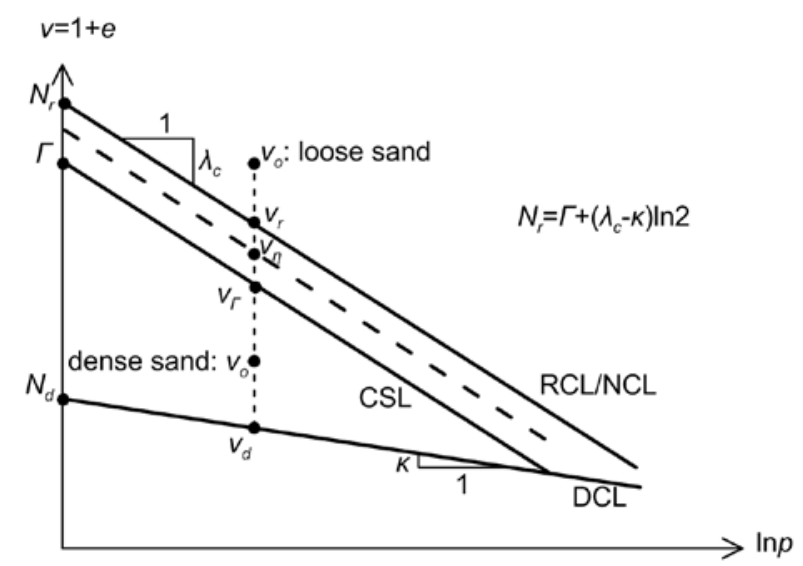

Figure 1. Dilatancy of sand with different initial densities in the $v$-Inp plane [27].

A state parameter $X_{1}$ is introduced to characterize the relative distance between the current specific volume and the corresponding specific volume on RCL:

$$
\chi_{1}=\max \left(\frac{v_{\eta}-v}{v_{r}-v_{d}}, 0\right)
$$

where $v$ is the current specific volume, $v_{r}$ is the specific volume on the RCL under the same mean effective stress (Figure 2), $v_{d}$ is the specific volume on the DCL, and $v_{\eta}$ is the specific volume on a line which is equivalent to the RCL but with a stress ratio of $\eta=q / p$, where $q$ is the current deviator stress. The state parameter $X_{1}$ becomes zero if the specific volume of the soil is on or above the RCL, but becomes equal to $X_{1}=1$ if $v$ is on the DCL.

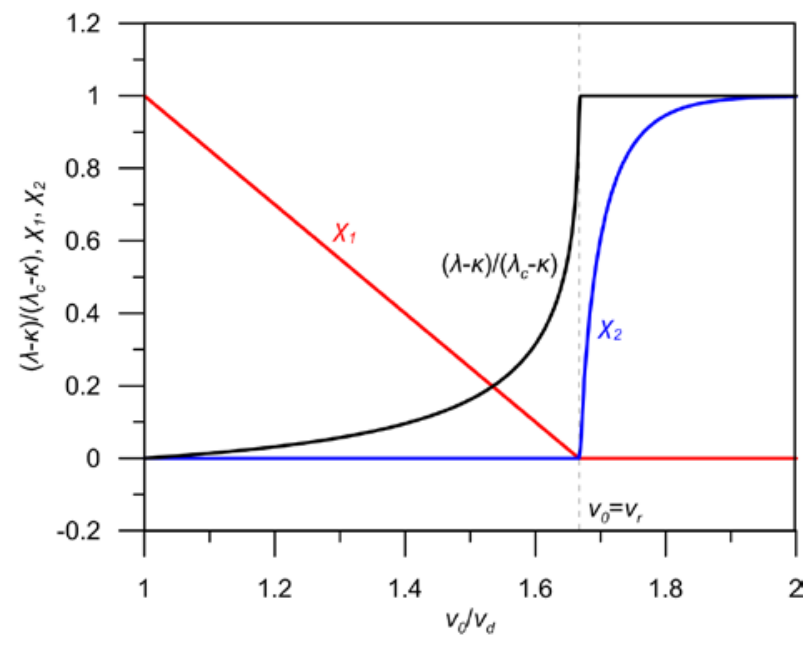

Figure 2. Variation of the state parameters and compressibility with initial specific volume, $v_{0}$. 
The peak shear strength of a soil depends on its relative location between the RCL and DCL. If its initial state is on or above the RCL, its peak shear strength slope is the same as the ultimate critical state slope $(M)$. If its initial state is on the DCL (the densest possible state), the peak shear strength slope reaches a maximum $\left(M_{\max }\right)$. For any states between the RCL and DCL, the peak shear strength slope $\left(M_{f}\right)$, varies between the slope $M$ of the CSL and the maximum possible slope $M_{\max }$ for the densest possible state. Here we assume the following interpolation holds:

$$
M_{f}=\left(M_{\max }-M\right) \sqrt{\chi_{1}}+M
$$

Eq. (2) suggests that $M_{f}$ becomes equal to $M$ when $X_{1}=0$ for an initial state on or above the RCL and $M_{\max }$ when $X_{1}=1$ for an initial state on the DCL.

In the MCC model, a soil with an initial specific volume below the NCL (or RCL) behaves purely elastically until the mean stress reaches the preconsolidation pressure. However, the model discussed here does not feature a purely elastic region. Instead, a soil with an initial state below the $\mathrm{RCL}$ will also undergo elastoplastic deformation under loading, with its compressibility depending on its current specific volume. In this context, we further assume that the compressibility of a soil under isotropic compression varies between the maximum slope $\lambda_{c}$ of the RCL and the minimum slope $k$ of the DCL, as:

$$
\frac{\lambda-\kappa}{\lambda_{c}-\kappa}=\frac{M^{4}}{M_{f}^{4}} \frac{M_{\max }^{4}-M_{f}^{4}}{M_{\max }^{4}-M^{4}}
$$

where $\lambda$ is the slope of the $v$-Inp line for any specific volume. It is clear that $\lambda$ equals $\lambda_{c}$ when the specific volume is on or above the RCL, but approaches $k$ when the specific volume is on the DCL. Note that $k$ also represents the slope of the unloading line (ULL), but not the slope of the reloading line. Any reloading in this model will cause plastic deformation and its slope varies between $\lambda_{c}$ and $K$ according to eq. (3). The variation of the slope of the reloading line with the specific volume implies that some residual volumetric strain occurs after one cycle of loading, unloading and subsequent reloading, as shown in Figure 3. However, no hysteretic loop occurs in the volumetric strain and the residual volumetric strain decreases with the number of cycles of loading and unloading (Figure 3.) The dependency of the slope of the compression line on the initial state is a concept used in other advanced models such as the bounding surface [14,15] or generalized plasticity models [16]. Such a model is of course not ideal for predicting the behavior of soils under cyclic loads, but would provide a first approximation.

Eq. (3) is only valid for isotropic compression. For anisotropic compression with a stress ratio of $\eta=q / p$, the equation becomes:

$$
\frac{\lambda-\kappa}{\lambda_{c}-\kappa}=\frac{M^{4}-\eta^{4}}{M_{f}^{4}-\eta^{4}} \frac{M_{\max }^{4}-M_{f}^{4}}{M_{\max }^{4}-M^{4}}
$$

Note that eq. (4) cannot be used to derive the slope of the CSL in $v$-Inp space by simply setting $\eta=M$, for a stress path may cross the CSL more than once as in conventional triaxial tests. The first time the stress path crosses the CSL usually corresponds to the phase transformation point where volume contraction changes to volume dilation. When the stress path eventually approaches the CSL, $M_{f}$ also approaches $M$ and therefore $\lambda$ approaches $\lambda_{c}$. The variation of the compressibility and the state parameter $X_{1}$ are plotted as functions of the initial specific volume in Figure 2. 


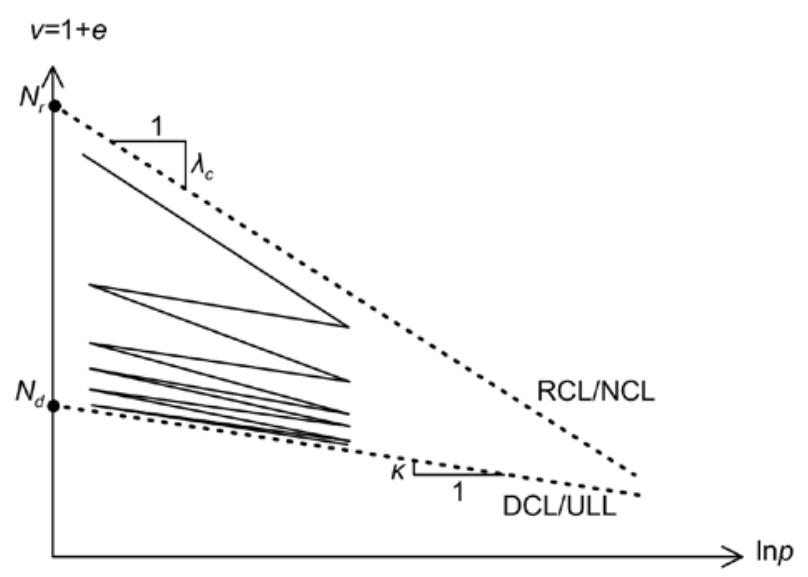

Figure 3. Variation of slope $\lambda$ under cyclic loading and unloading.

\subsection{Yield function and plastic potential}

The yield function of the model is similar to that of the MCC model. An additional parameter, $X_{2}$, is introduced so that the yield surface changes from ellipse to teardrop shape when $X_{2}$ changes from zero to 1 , as illustrated in Figure 4 . This change of the yield surface shape is necessary to capture the undrained behavior of very loose sands and will be demonstrated later. The modified yield function takes the form:

$f=q^{2}-\frac{p^{2}\left(p_{c}-p\right)}{p+\chi_{2}\left(p_{c}-p\right)} M^{2}=0$

where $p_{c}$ is the yield surface size along the $p$ axis, and $\chi_{2}$ is a state parameter that depends on the initial void ratio of the soil. Note that eq. (5) cannot be interpreted as the boundary of the elastic region because, as mentioned above, there is no such purely elastic region in this model. Any initial stress state is always located on the yield surface, regardless whether the soil is normally consolidated or overconsolidated. In addition, the yield surface has a vertex at the origin with $X_{2}>0$, and this is a singularity point where the gradients of the yield function do not exist. This singularity can be eliminated by smoothing the yield surface around the origin.

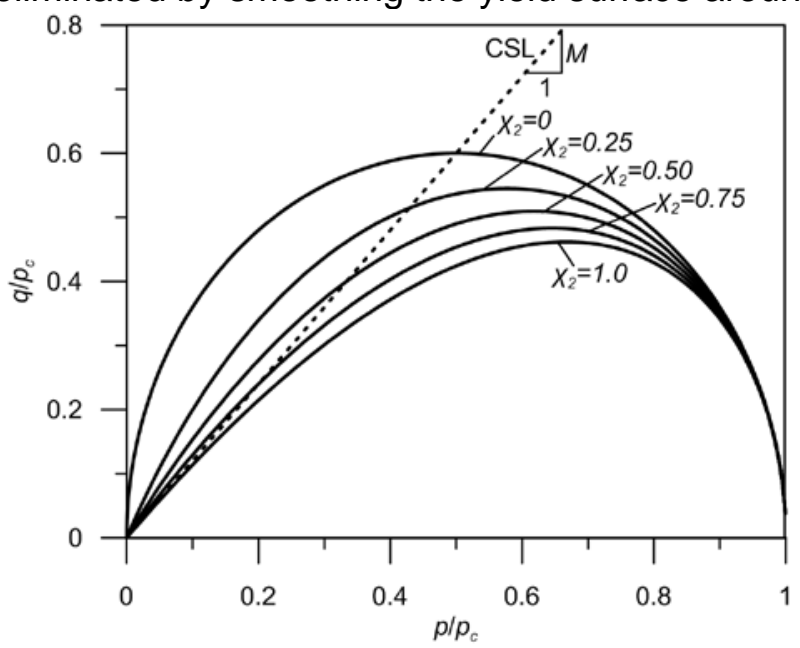

Figure 4. Variation of yield surface according to state parameter $X_{2}$ for $M=1.2$.

The state parameter $X_{2}$ represents a relative difference between the initial specific volume and the corresponding critical state specific volume (Figure 1). For the given yield function of eq. (5), the distance between the initial specific volume and the critical state specific volume can be found by following a stress path where the soil is 
first isotropically loaded from mean stress $p_{c} / 2$ to $p_{c}$ and then unloaded anisotropically to stress state $\left(p_{c} / 2, M p_{c} / 2\right)$ on the CSL:

$$
\begin{aligned}
& v_{0}-v_{1}=\left(\lambda_{c}-\kappa\right) \ln \left(1+\frac{1}{1-\chi_{2}}\right) \\
& \chi_{2}=\max \left(\frac{\exp \left(\frac{v_{0}-v_{\Gamma}}{\lambda_{c}-\kappa}\right)-2}{\exp \left(\frac{v_{0}-v_{\Gamma}}{\lambda_{c}-\kappa}\right)-1}, 0\right)=\max \left(\frac{\exp \left(\frac{v_{0}-v_{r}}{\lambda_{c}-\kappa}\right)}{\exp \left(\frac{v_{0}-v_{\Gamma}}{\lambda_{c}-\kappa}\right)-1}, 0\right)
\end{aligned}
$$

where $v_{0}$ is the initial specific volume and $v_{\Gamma}$ is the specific volume on the CSL under the same mean effective stress (Figure 1). It is clear that $X_{2}$ approaches zero once the initial specific volume is on or below the RCL, and approaches asymptotically $X_{2}=1$ if $v_{0}$ tends to infinity. The variation of $X_{2}$ with the initial specific volume is illustrated in Figure 2.

In addition to the above, the plastic potential function is assumed to be the same as that in the MCC model:

$$
g=q^{2}-M^{2} p\left(p_{c}-p\right)
$$

\subsection{Hardening law}

The hardening law follows that of the MCC model where the plastic volumetric strain, $\varepsilon_{v}^{p}$, is considered as the only hardening parameter:

$$
\mathrm{d} p_{c}=\frac{v p_{c}}{\lambda-\kappa} \mathrm{d} \varepsilon_{v}^{p}=\frac{v p_{c}}{\lambda_{c}-\kappa} \frac{M_{\max }^{4}-M^{4}}{M_{\max }^{4}-M_{f}^{4}} \frac{M_{f}^{4}-\eta^{4}}{M^{4}-\eta^{4}} \mathrm{~d} \varepsilon_{v}^{p}
$$

Using the flow rule $\mathrm{d} \varepsilon_{v}^{p}=\dot{\Lambda} \partial / \partial p$ and the yield function (eq. 5), we can eliminate the singularity point in eq. (8) when $\eta=M$ by re-writing the hardening law as:

$$
\mathrm{d} p_{c}= \begin{cases}\frac{v p^{2}}{\lambda_{c}-\kappa} \frac{M_{\max }^{4}-M_{\max }^{4}-M_{f}^{4}}{M_{f}^{4}-\eta^{4}} \dot{\Lambda} & v_{0}<v_{r} \\ \frac{v p_{c}}{\lambda_{c}-\kappa} M^{2}\left(2 p-p_{c}\right) \dot{\Lambda} & v_{0} \geq v_{r}\end{cases}
$$

where $\dot{\Lambda}$ is the plastic multiplier and is strictly non-negative. The hardening law above can result in both hardening $\left(\mathrm{d} p_{c}>0\right)$ and softening $\left(\mathrm{d} p_{c}<0\right)$, depending on the relative magnitude of $M_{f}$ and $\eta$ or the relative magnitude of $p$ and $p_{c}$.

\subsection{Three-dimensional stress states}

All the functions presented above are for triaxial stress states. These functions can be generalised to 3-D stress states through the $g(\theta)$ method. In the $g(\theta)$ method, the slope $M$ as well as $M_{f}$ and $M_{\max }$ is expressed as a function of Lode angle $\theta$, as in Sheng et al. [28]: 


$$
M(\theta)=M\left(\frac{2 \alpha^{4}}{1+\alpha^{4}-\left(1-\alpha^{4}\right) \sin 3 \theta}\right)^{1 / 4}
$$

where $\theta$ is the Lode angle, $M$ is the slope of the CSL under triaxial compression stress states and $\alpha=3 /(3+M)$. The function described in eq. (10) ensures the convexity of the critical state surface in the deviatoric plane for any friction angle less than $48.6^{\circ}$ (or $M<2$ ). The same function applies to the slopes $M_{f}$ and $M_{\max }$.

\subsection{Model Parameters}

There are all together 7 material parameters in the model:

1. The critical state friction angle of the soil, $\varphi_{c r}$ (or the slope of CSL in $p-q$ space under triaxial compression, $M$ )

2. The maximum peak shear strength angle, $\varphi_{\max }$ (or the maximum possible shear strength under triaxial compression, $M_{\max }$ )

3. The slope of the critical state line in $v$-Inp space, $\lambda_{c}$

4. The slope of unloading line in $v$-Inp space, $K$

5. The specific volume on the RCL when $p=1 \mathrm{kPa}, N_{r}$

6. The specific volume on the DCL when $p=1 \mathrm{kPa}, N_{d}$

7. The Poisson ratio

The state parameter $X_{2}$ depends only on the initial specific volume, whereas the state parameter $X_{1}$ depends on the current specific volume as well as the current stress state. Note that the two state parameters $X_{1}$ and $X_{2}$ introduced here do not follow the definition of the state parameters in Yao et al. [27].

\subsection{Sand behaviour in triaxial compression tests}

In the following Figures 5 and 6 , we compendiously present and accordingly assess model predictions in conceptual triaxial compression tests; emphasis on the model performance is put on its comparison with centrifuge testing results in the boundary value problem described in the following sections. The indicative model parameters consider are listed in Table 1 and are selected so as to be compatible with the model parameters employed by Yao et al. [27] for Toyoura sand.

Table 1. Indicative constitutive model parameters used to obtain model predictions in conceptual triaxial tests.

\begin{tabular}{|c|c|c|c|c|c|c|}
\hline$\lambda_{c}$ & $K$ & $N_{r}$ & $N_{d}$ & $\varphi_{c r}$ & $\varphi_{\max }$ & Poisson ratio \\
\hline 0.09 & 0.02 & 2.45 & 1.70 & $26.6^{\circ}$ & $47^{\circ}$ & 0.33 \\
\hline
\end{tabular}

The density-dependent peak shear strength and volume change behavior of sand are well captured by the model. The three dense sand states with initial specific volumes $v_{0}=1.65 \sim 1.86$ all exhibit a peak shear strength and volume dilation in the drained tests (Figure 5). Unlike the MCC model, the deviator stress approaches the peak values smoothly, and the phase transition where volume contraction changes to volume dilation occurs before the peak deviator stresses. The magnitudes of the peak shear stresses and the volume dilation depend on the initial density of the sand. In the undrained triaxial tests (Figure 6), the shear strength again is densitydependent. A very loose sand with a specific volume of $v_{0}=2.05$ would exhibit a tendency to static liquefaction, with the deviator stress approaching zero as shearing continues. On the other hand, the dense sands with specific volumes $v_{0}=1.86$ and $v_{0}=1.76$ show a tendency of phase transition from contraction (positive excess pore pressure) to dilation (negative excess pore pressure). These generic results are generally in agreement with sand behavior observed in triaxial tests. While the 
enhanced model is significantly simpler than the original model by Yao et al. [27], the predicted results in Figures 5 and 6 compare well with those presented in Yao et al. [27]. Some differences are noted however when comparing model predictions with Yao et al. [27] predictions in undrained test results: the peak shear stresses for the dense sands $\left(v_{0}=1.76 \sim 1.89\right)$ obtained with the enhanced model presented herein are somewhat lower than those predicted by the original model.
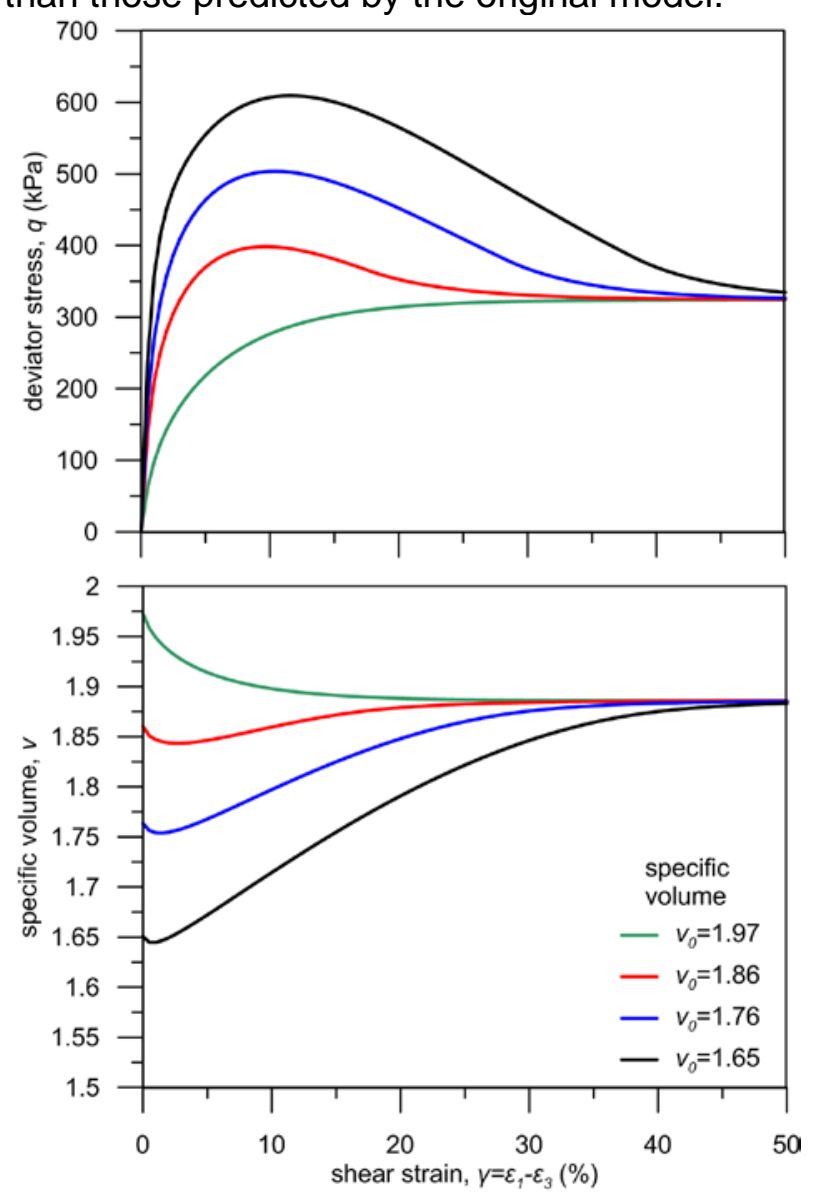

Figure 5. Drained conceptual triaxial compression tests on dense and loose sands.

\subsection{Stress integration and implementation in ABAQUS/Explicit}

The model has been implemented in the finite element code ABAQUS/Explicit through the user subroutine VUMAT, in vectorized format [21]. The explicit calculation selected to solve the boundary value problem has many capabilities in performing large deformation simulations, including an Arbitrary Langragian-Eulerian (ALE) remeshing technique to preserve mesh quality, and the ability to robustly account for friction at the cone-soil interface. Due to the relative simplicity of the constitutive model, an explicit stress integration scheme that features automatic substepping and error control is used to solve the incremental stress-strain relation $[29,30]$. In fact, a key complication of the explicit scheme, namely the algorithm used to find the intersection between the elastic trial stress path and the current yield surface, is not necessary for the particular model, as loading is always treated as elastoplastic (the current yield surface always goes through the current stress point). 

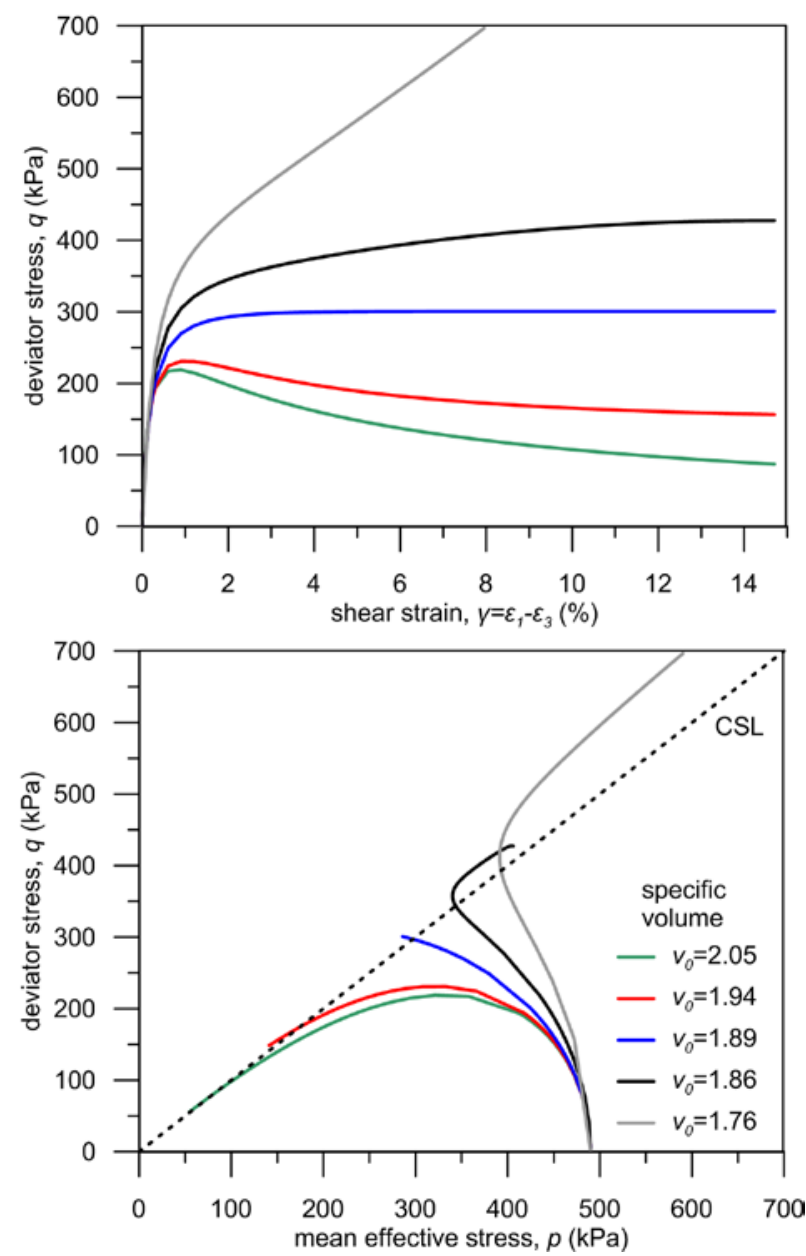

Figure 6. Undrained conceptual triaxial compression tests on dense and loose sands.

\section{Centrifuge test Results CONSIDERED FOR the CALIBRATION AND VALIDATION OF THE NUMERICAL METHODOLOGY}

In order to validate the efficiency of the proposed constitutive model in simulating cone penetration testing in dry sand of varying density, we will replicate the $70 \mathrm{~g}$ centrifuge tests carried out by Bolton and Gui [22] at the Cambridge Geotechnical Centrifuge Center. The cone penetration experiments were performed in dry Fontainbleau sand (Table 2), using a $10 \mathrm{~mm}$ diameter cone. $D_{50}$ is the grain diameter at which $50 \%$ of the soil is finer. Bolton and Gui's study focused on the effect of the relative density of the sand and of the container dimensions on the cone penetration resistance; no sleeve friction measurements were obtained during the tests, as sand density is correlated with the cone resistance only.

Table 2. Properties of the Fontainbleau sand reported by Bolton and Gui [22].

\begin{tabular}{|l|c|}
\hline Grain diameter $D_{50}(\mathrm{~mm})$ & 0.181 \\
\hline Coefficient of uniformity, $C_{u}$ & 1.69 \\
\hline Maximum void ratio, $e_{\max }$ & 0.92 \\
\hline Minimum void ratio, $e_{\min }$ & 0.55 \\
\hline Specific gravity, $G_{s}$ & 2.644 \\
\hline
\end{tabular}

Details on the model preparation method and testing are not presented here. Instead, we focus on the results of experiments MWG5, MWG12, MWG9 and MWG10, which were back-analyzed to calibrate the constitutive model parameters. The former two were performed in very dense sand $\left(D_{r}=89 \%\right)$ and the latter two in medium sand $\left(D_{r}=54 \%\right)$, in containers of different sizes (Table 3$)$. In addition to these 
four experiments, we present for comparison purposes two additional experiments; MWG8 performed in medium sand $\left(D_{r}=58 \%\right)$, and MWG11 performed in very dense sand $\left(D_{r}=76 \%\right)$.

Table 3. Centrifuge tests performed by Bolton and Gui [22].

\begin{tabular}{|l|c|c|c|c|c|c|}
\hline Test & MWG5 & MWG8 & MWG9 & MWG10 & MWG11 & MWG12 \\
\hline Diameter of container $(\mathrm{mm})$ & 850 & 850 & 850 & 210 & 210 & 210 \\
\hline Height of specimen $(\mathrm{mm})$ & 350 & 351 & 348 & 358 & 351 & 342 \\
\hline Dry density, $\rho\left(\mathrm{kg} / \mathrm{m}^{3}\right)$ & 1663 & 1552 & 1538 & 1538 & 1613 & 1662 \\
\hline Void ratio, $e$ & 0.590 & 0.704 & 0.719 & 0.719 & 0.639 & 0.590 \\
\hline Relative density, $D_{r}(\%)$ & 89 & 58 & 54 & 54 & 76 & 89 \\
\hline Rate of penetration $(\mathrm{mm} / \mathrm{sec})$ & 3.6 & 3.5 & 3.5 & 3.7 & 3.7 & 3.7 \\
\hline
\end{tabular}

Further evidence on the validity of the presented numerical methodology will be sought from the comparison with similar cone penetration test results from five independent laboratories, reported by Bolton et al. [7]. These tests were performed in the same Fontainbleau sand of average relative density $\left(D_{r}=84 \%\right)$, but using different preparation methods, container sizes and cone diameters. Bolton et al. [7] reported "very encouraging" repeatability of CPT simulation in centrifuge tests from different laboratories, underlining the reliability of these measurements as reference tests for comparison purposes. It must be noted that the cone diameters used in the independent tests ranged between $d=10 \mathrm{~mm}$ to $d=12 \mathrm{~mm}$. Bolton et al. [7] commented that, in order to achieve "unbiased results", the cone diameter $d$ must be at least twenty times greater than the mean particle diameter $D_{50}$. Senders [31] on the other hand suggested that the cone resistance depends on the probe diameter, and proposed a formula to account for the effect of the cone size on the cone resistance, although his method is not verified against experimental measurements. Nevertheless, the present numerical method can be used to simulate penetration of probes of different sizes, as the geometry of the cone is taken into account during the calibration against the benchmark experimental results.

\section{DESCRIPTION AND VALIDATION OF THE NUMERICAL MODEL}

The finite element model was developed with the aim to replicate Bolton and Gui [22] test MWG5, MWG12, MWG9 and MWG10 results, in terms of the resistance developing on the cone with the depth of penetration. The cone model in centrifuge, rather than the prototype of cone, was reproduced. The properties of the model presented in the following resulted from an extensive numerical study, investigating the effects on the results, run times, and the numerical stability of the code of several parameters, including: the extents of the simulated geometry; the finite element type, size and mesh grading; simulation of the penetration procedure and surface properties; constitutive model integration parameters; adaptive meshing technique and controls; etc. Details on this study are omitted, to preserve the length of the presentation. However, the parameters of the model that were finally adopted will be outlined here, as they may be of interest for readers engaged with the numerical simulation of similar problems.

The problem of the cone penetration was simulated as axisymmetric, in model scale, considering a geometry of $0.14 \mathrm{~m} \times 0.35 \mathrm{~m}$ (Figure 7). Loukidis and Salgado [32] mention that in order to restrain the shear band thickness next to the cone shaft to realistic values, when performing numerical analyses with a strain-softening material, the minimum element size should be between 5 to $20 D_{50}$. This suggestion is based on the experimental work on shear strain localization by Uesugi et al. [33], Vardoulakis and Sulem [34] and Nemat-Naser and Okada [35]. Grain diameter $D_{50}$ for the Fontainbleau sand is $D_{50}=0.181 \mathrm{~mm}$ (Table 2), and the element size around the penetrometer was $2 \mathrm{~mm}\left(11 D_{50}\right)$, thus within the acceptable range. The ratio of the soil container over the cone diameter was 28 (Figure 7). 


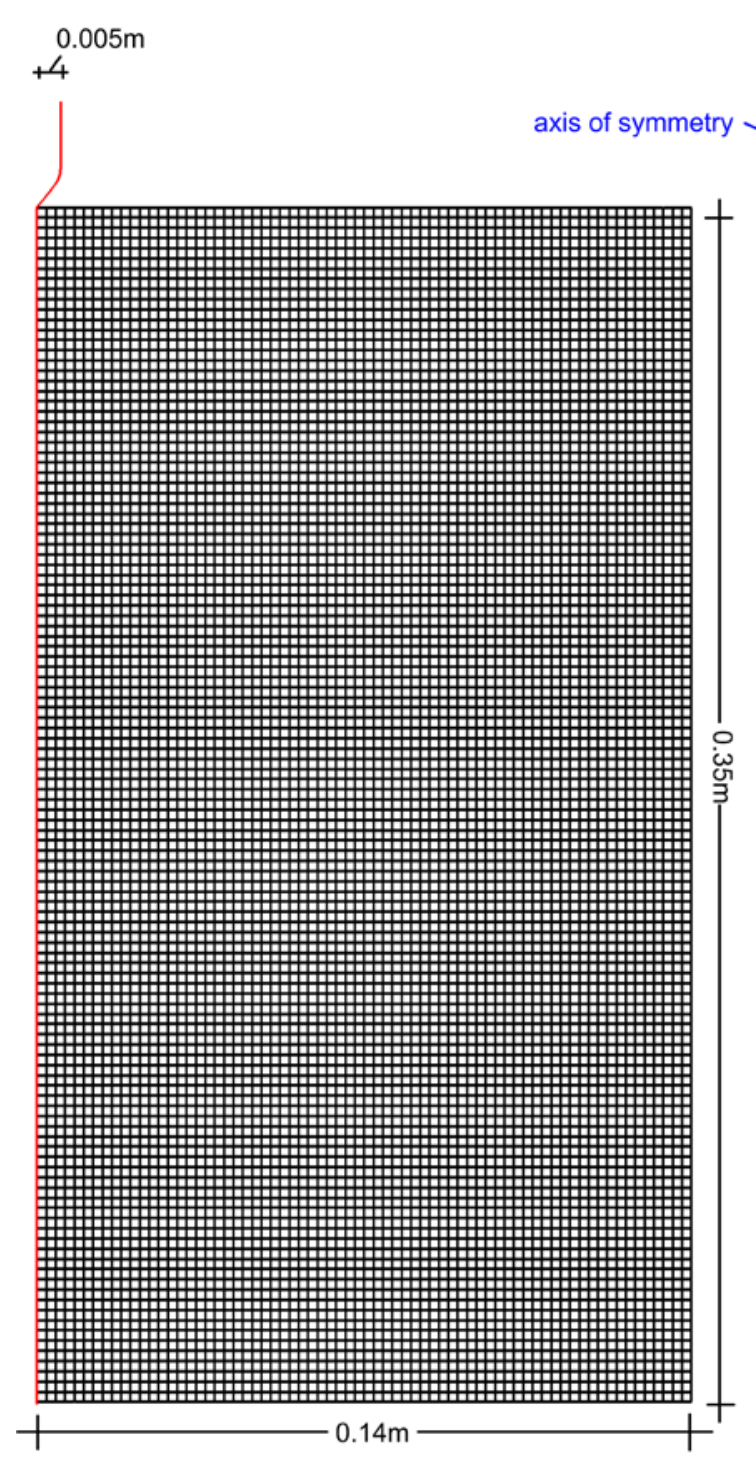

(a)

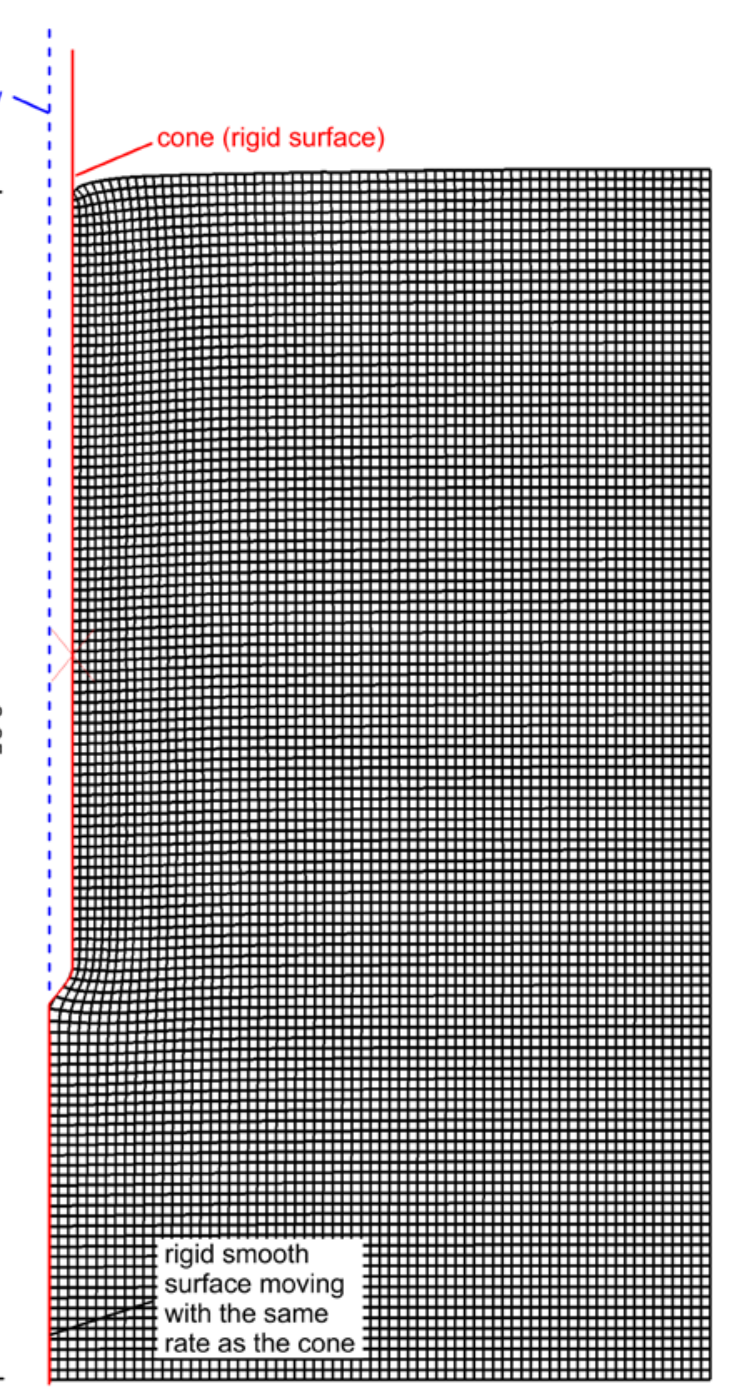

(b)

Figure 7. (a) Finite element mesh employed in the analyses, and (b) Deformed finite element mesh for cone penetration 25D.

A total of 8190 4-node reduced-integration elements (CAX4R) were used to simulate the sand inside the container. The "combined" viscous-stiffness hourglass control option was used, together with the second-order accuracy formulation. The cone was modeled as a Lagrangian rigid surface with a diameter $d=10 \mathrm{~mm}$ [22] and a standard apex angle $60^{\circ}$. Penetration of the cone into the sand is simulated by prescribing a vertical displacement on a reference node, and soil along the axis of symmetry is allowed to move laterally to accommodate the deformation imposed by cone penetration (Figure 7). Various numerical techniques have been proposed in the literature to accommodate this issue, such as slightly offsetting the left boundary from axis of symmetry or defining a pre-existing cavity underneath the cone tip [36, 37, 38]. However, a different, perhaps more robust technique was introduced in this study, resulting in enhanced mesh quality: a smooth rigid surface was defined, originating below the tip of the cone and extending below the bottom boundary of the model (Figure 7b). This surface is moving together with the cone, thus preventing the leftmost soil elements to move inwards of the axis of symmetry but not outwards; therefore allowing the cone surface to slide.

The problem was simulated dynamically in ABAQUS/Explicit, with a slow penetration rate of $0.25 \mathrm{~mm} / \mathrm{sec}$, to avoid numerical issues related to the sudden application of 
high strain rates, and inertia effects. This rate was about 10 times slower than the actual rate of the tests (Table 3 ). The total penetration of the cone tip reached $0.25 \mathrm{~m}$ in model scale i.e. 25 times the cone diameter.

As the use of even small values of either fixed or variable mass scaling (a common technique to artificially increase the timestep in quasi-static analyses) proved to have an effect on the analysis results, another method was used to accelerate the solution to acceptable levels: Instead of applying a gravitational force, the geostatic stress field was defined via a body force acting on the soil elements, taking advantage of the geometry of the problem. The applied body force magnitude is $B=\rho \cdot g \cdot N$ where $\rho$ is the soil density, and $N$ is test acceleration level in g's, here $N=70$. Thus geostatic effective stresses are equal to the centrifuge model, while horizontal stresses were calculated by considering the earth pressure coefficient at rest equal to $K_{0}=0.5$. Not including a gravitational force (geostatic stresses do not depend on density) allowed for increasing the soil density by 10,000 times, and a subsequent increase in the minimum time step while keeping the penetration rate slow and avoiding the development of compressional waves that would be reflected on the fixed boundaries. Consequently, each full analysis required about $10^{6}$ steps, and took 50 70 hours to complete in a 4X6 core Xeon CPU's server.

To minimize element distortion during cone penetration into the sand, the ALE adaptive mesh option was employed. Although this involves a high computational cost, maintaining the quality of the grid and avoiding excessive element distortion required regeneration of the mesh after each single analysis step (Figure $7 b$ ). In addition, the curvature refinement parameter $a_{c}$ [21] was set equal to zero, to maintain the desired element size and shape along the inteface of the sand with the concave surface representing the penetrating cone.

Friction at the interface between the cone and the sand was simulated with the classical Coulomb friction law. The friction coefficient at the cone-sand interface was assumed constant, and was determined from the back-analysis of centrifuge test results equal to $\mu=0.5$. Note that simulating the cone tip and probe as rigid surface allows for the estimation of the CPT sleeve friction from the numerical analyses: we can compute the vertical reaction on a "friction sleeve", defined as a finite part of the vertical surface representing the probe. Subsequently the friction ratio can be calculated, equal to the sleeve friction resistance over the cone tip resistance, which is used to determine the soil stratigraphy from cone penetration tests. Assuming here a friction coefficient equal to $\mu=0.5$ resulted in a friction ratio of the order of $0.4-0.5 \%$, typical of a clean sand.

Calibration of the constitutive model parameters (Table 4) was too based on the back-analysis of centrifuge test results, via two benchmark analyses for medium sand (relative density $D_{r}=54 \%$, initial specific volume $v_{0}=1.72$ ) and very dense sand (relative density $D_{r}=89 \%$, initial specific volume $v_{0}=1.59$ ).

Table 4. Constitutive model parameters used in the numerical analyses.

\begin{tabular}{|c|c|c|c|c|c|c|}
\hline$\lambda_{c}$ & $\kappa$ & $N_{r}$ & $N_{d}$ & $\varphi_{c r}$ & $\varphi_{\max }$ & Poisson ratio \\
\hline 0.0085 & 0.006 & 1.89 & 1.58 & $30^{\circ}$ & $50^{\circ}$ & 0.333 \\
\hline
\end{tabular}

Centrifuge tests measurements are presented in terms of cone resistance $q_{c}$ versus the corrected prototype penetration depth, $z_{p c}[7,39]$. The corrected prototype depth is defined as:

$$
z_{p c}=z_{p}\left[1+\left(\frac{z_{m}}{2 R}\right)\right]
$$


where $z_{p}$ is the prototype penetration depth, $z_{m}$ is the model penetration depth, and $R=0.3755 \mathrm{~m}$ is the average radius to the surface of the sand specimen, measured from the central axis of the centrifuge rig [7]. In the above equation it is:

$$
z_{p}=N \cdot z_{m}
$$

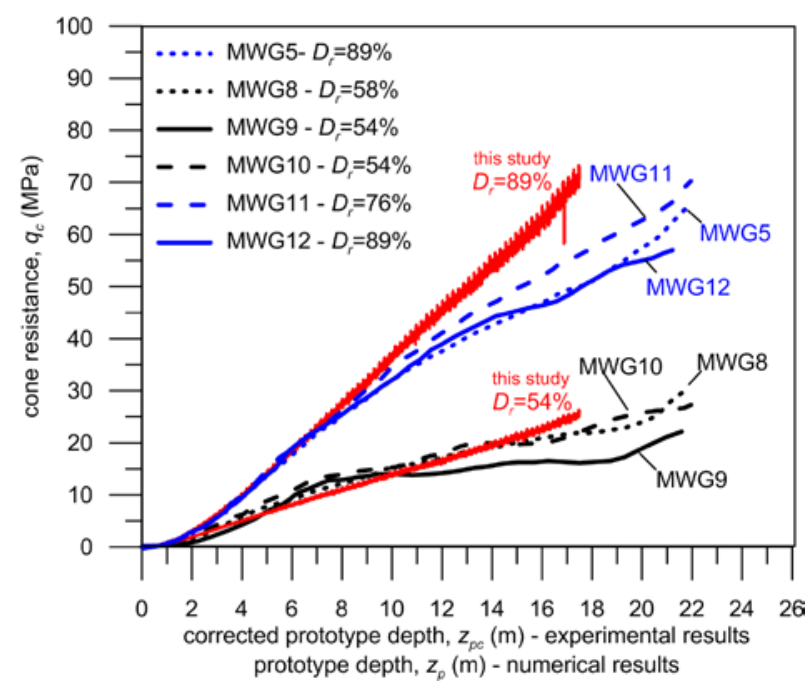

Figure 8. Comparison of the results of the basic numerical analyses employed for the calibration of the model, with the tests performed by Bolton and Gui [22].

Results of the two benchmark numerical analyses mentioned earlier for medium sand and very dense sand are depicted in Figure 8, together with the results from the centrifuge tests mentioned in Table 3. A fair agreement with the centrifuge test measurements is observed, irrespective of the sand density and penetration depth. Note that the numerical cone resistance values are plotted against the prototype penetration depth, $z_{p}$ (eq. 12). Numerical cone resistance curves inevitably include some noise, due to the frequent remeshing, yet we decided not to filter it in order to present its effect on the practical results of the analyses. Further observe in Figure 9 that, as expected, the zone of sand disturbed by cone penetration depends on the initial relative density of the sand. In the case of medium sand (Figure 9a) loosening is noticed around the cone tip while above, and secondarily below it, the sand is densified due to cone penetration. On the contrary, loosening of the sand is noticed around the cone during penetration in dense sand (Figure 9b), and a greater lateral zone of about two cone diameters is affected. 


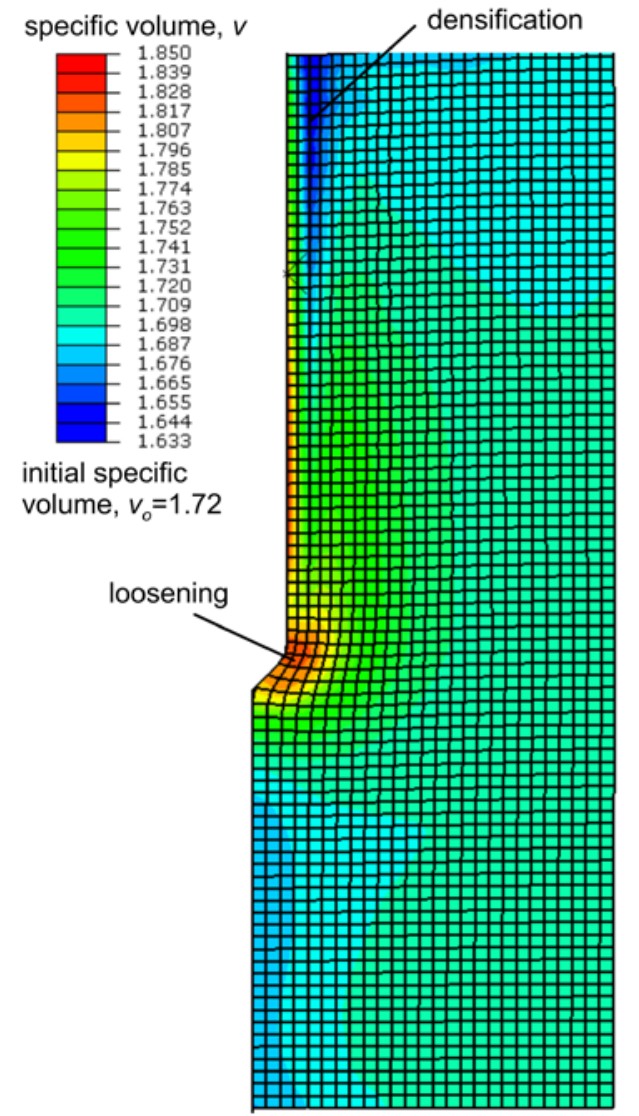

(a)

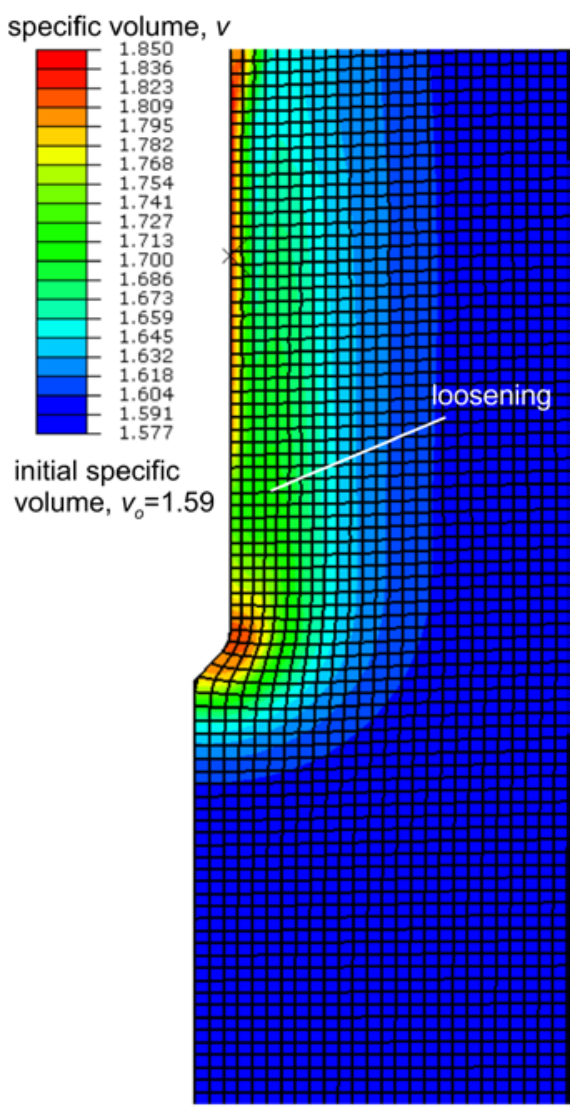

(b)

Figure 9. Specific volume contours in the vicinity of the cone for (a) $D_{r}=54 \%$ and (b) $D_{r}=89 \%$ (detail).

Results of an additional analysis considering dense sand (relative density $D_{r}=84 \%$, initial specific volume $v_{0}=1.61$ ) are compared in Figure 10 to the results of the centrifuge tests from five independent laboratories reported by Bolton et al. [7]. Results are also compared in terms of the normalize cone resistance, $Q$, to be in line with Bolton et al. [7] presentation:
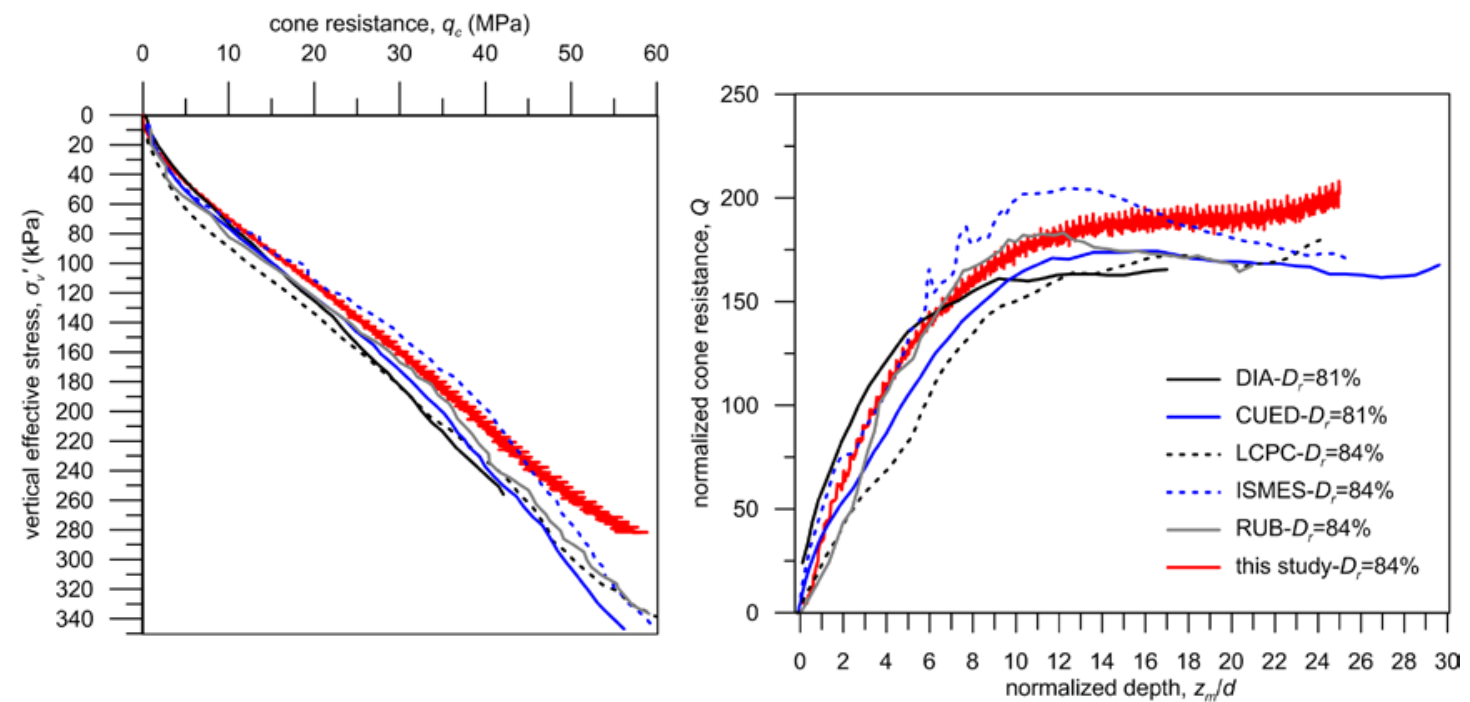

Figure 10. Comparison of the results of the numerical analysis for $D_{r}=84 \%$ with the test results from five laboratories: Technical University of Denmark (DIA), Cambridge University Engineering Department (CUED), Laboratoire Central des Ponts et Chaussées (LCPC), Istituto Sperimentale Modelli e Strutture (ISMES) and RuhrUniversität (RUB); as reported by Bolton et al. [7]. 


$$
Q=\frac{q_{c}-\sigma_{v}}{\sigma_{v}^{\prime}}
$$

where for the dry sand it is:

$$
\begin{array}{ll}
\sigma_{v}^{\prime}=\sigma_{v}=\rho \cdot g \cdot z_{p}\left[1+\left(\frac{z_{m}}{2 R}\right)\right] & \text { for the centrifuge tests } \\
\sigma_{v}^{\prime}=\sigma_{v}=\rho \cdot g \cdot z_{p} & \text { for the numerical analyses }
\end{array}
$$

Observe that numerical results are consistent with the reference centrifuge experiments; whereas the shape of the normalized cone resistance curve suggests that the model is able to effectively capture cone-sand interaction effects both above and below the transition depth from shallow to deep foundation mechanism, where the normalized resistance $Q$ remains generally constant with depth [7].

\section{Parametric investigation of SAND State efFects on the cone PENETRATION RESISTANCE}

One of the main aims of Bolton and Gui [22] experimental study was the correlation of the relative density of the sand with the cone tip resistance. Bolton and Gui [22] suggested that the relation between the sand relative density and the maximum normalized cone resistance $Q$ could be approached with a curve similar to the dashed one in Figure 11, which was "not determinable" due to the lack of additional test data. Instead, Bolton and Gui proposed a linear expression (solid black line in Figure 11)

$D_{r}(\%)=0.2831 \cdot Q+32.964$

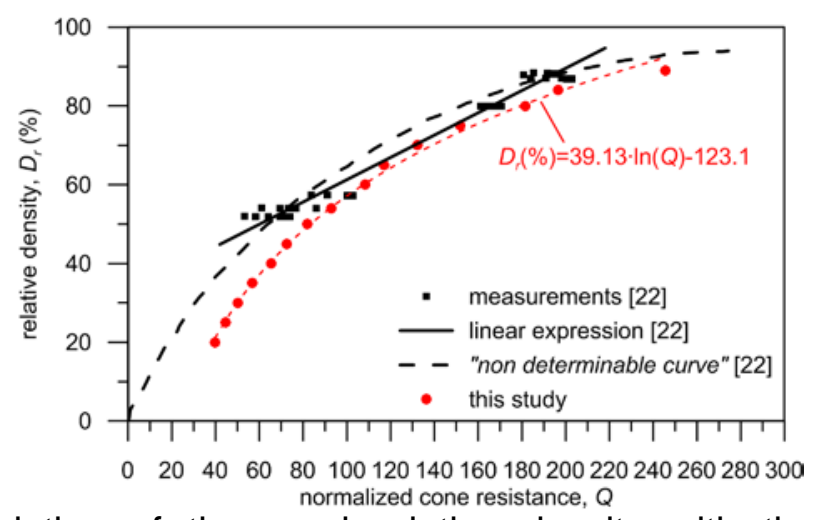

Figure 11. Correlation of the sand relative density with the normalized cone resistance. The results of the parametric numerical analyses are presented against the experimental results reported by Bolton and Gui [22], and the linear correlation that they proposed.

During this study, 15 additional parametric analyses were executed to fill in the gap in Bolton and Gui's measurements, considering sand relative densities within the range $D_{r}=20 \%$ and $D_{r}=90 \%$, commonly met in practice. If we plot the maximum normalized tip resistance derived from these analyses against the relative density (Figure 11), we can define the "non determinable" curve discussed by Bolton and Gui. Perhaps not surprisingly, the curve resulting from the parametric numerical analyses matches well with the hypothetical curve drawn by Bolton and Gui [22]. We can further proceed with the proposal of a curve fitting expression, to provide the relative density of the Fontainbleau sand as a function of the normalized cone tip resistance: 
$D_{r}(\%)=39.13 \cdot \ln (Q)-123.1$

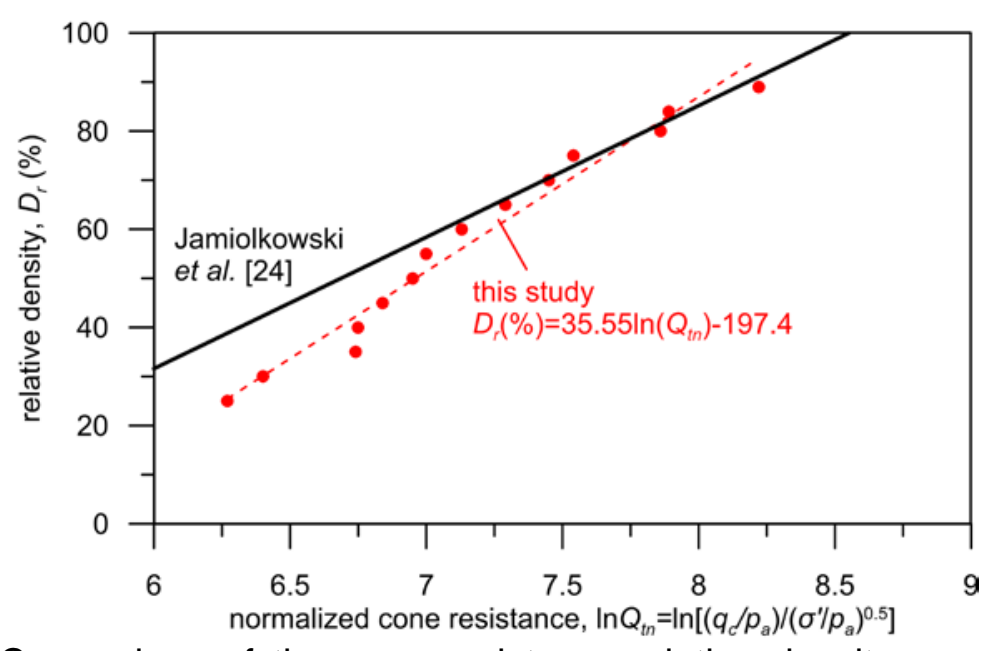

Figure 12. Comparison of the cone resistance-relative density correlation derived from the parametric analyses with the correlation proposed by Jamiolkowksi et al. [24].

Results of this parametric numerical study are plotted again in Figure 12, in comparison to the well-established formula by Jamiolkowksi et al. [24], which resulted from the interpretation of a large number of calibration chamber test results from different sands, corrected to account for the chamber size [40]:

$D_{r}(\%)=26.8 \cdot \ln \left(Q_{t n}\right)-129.2$

where

$Q_{t n}=\left(q_{c} / p_{a}\right) /\left(\sigma_{v}^{\prime} / p_{a}\right)^{m}$

and $p_{a}$ is the atmospheric pressure, in the same unit system of the cone penetration resistance and vertical stress. According to Robertson [41] the exponent $m$ in eq. (18) is closer to 0.5 for clean sands, and closer to 1.0 for clays. If we employ the stress normalization factor proposed by Jamiolkowksi et al. [24] i.e. $m=0.5$, eq. (16) is transformed as:

$D_{r}(\%)=35.55 \cdot \ln \left(Q_{t n}\right)-197.4$

Notice from Figure 12 that the expression proposed by Jamiolkowksi et al. matches fairly well the numerical results and the corresponding fit expression from this study, at least for medium- to dense sands with $D_{r}>50 \%$.

Further elaboration on the results of the parametric "numerical CPT tests" can lead to the correlation of the cone resistance to the density-related state parameter $\psi[23$, 42], that introduces the effect of stress level on the sand state:

$\psi=e-e_{\Gamma}=v-v_{\Gamma}$

where

$v_{\Gamma}=\Gamma-\lambda_{c} \ln p$

and $\Gamma=N_{r^{-}}\left(\lambda_{c}-k\right) \ln 2$ (Figure 2). The mean stress under geostatic conditions is: 
$p=\sigma_{v}\left(\frac{1+2 K_{0}}{3}\right)$

with $K_{0}=0.5$ here. Jefferies and Been [42], based on the evaluation of a series of calibration chamber tests on NC and OC sands, proposed that $\psi$ can be estimated from CPT cone resistance measurements via an empirical expression with the form:

$$
Q_{p}=k \exp (-m \psi)
$$

where $Q_{p}=\left(q_{c}-p\right) / p$ the normalized cone resistance over the mean stress and $k, m$ are sand-specific constants. The above eq. (22) suggests that the constants $k$ and $m$ do not depend on the mean stress $p$, whereas Sladen [43] reports substantial bias of $\psi$ with the stress level. While Jefferies and Been [42] agree that such a stress bias exists, they argue that it leads to an uncertainty in the estimated state parameter value less than $|\Delta \psi|<0.05$. As depicted in Figure 13, numerically derived $\psi-p$ curves for different initial sand density suggest that indeed the state parameter depends on the mean stress, however this dependency becomes insignificant for mean stress levels higher than $p=50-100 \mathrm{kPa}$ i.e. when the deep foundation mechanism governs.

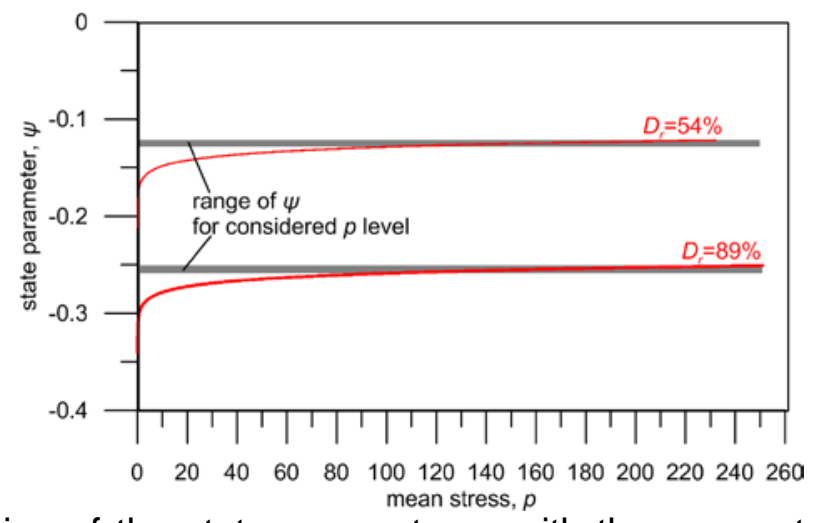

Figure 13. Evolution of the state parameter $\psi$ with the mean stress, $p$ for different sand densities.

Considering for simplicity mean stress levels higher than $p=100 \mathrm{kPa}$, where the stress bias effect becomes negligible, we can estimate the state parameter $\psi$ from the parametric numerical analyses as a function of the steady-state normalized cone resistance $Q_{p}$. Results of this interpretation are presented in Figure 14, and are compared against corresponding curves covering the range of expected $k$ and $m$ constant values for different sands [42]. The fitting curve applying to the results of this study lies within the range of cone resistance-state parameter curves drawn by Jefferies and Been [42] (which do not cover Fontainbleau sand). Attention should be paid however to the fact that the constants $k$ and $m$ depend strongly on the type of the sand, resulting in substantial scatter in state parameter estimations. 


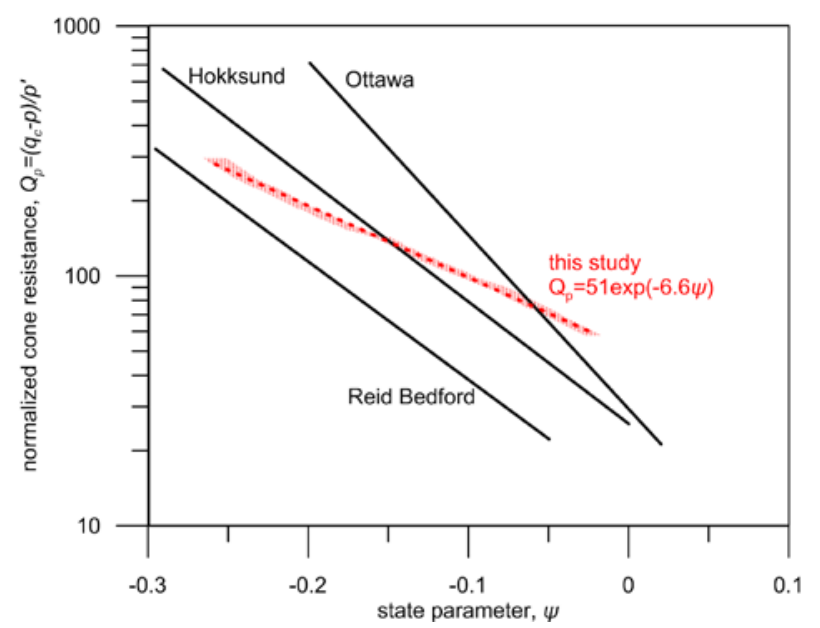

Figure 14. Comparison of the cone resistance-state parameter correlation derived from the parametric analyses with corresponding curves for different sands by Jefferies and Been [42]. The range of results from the numerical analyses, presented with red hatch, indicates the dependency of the state parameter on the stress level for $p>100 \mathrm{kPa}$.

\section{SUMMARY AND PRACTICAL REMARKS}

A numerical study on the simulation of cone penetration tests in sand was presented. It is based on a new, computationally effective, constitutive model, introduced for the description of loose-to-dense sand response in (very) large displacement problems, aiming at accurately accounting for the key parameters controlling cone resistance: sand relative density, stress level, and sand compressibility.

The model is implemented in vectorized format in the finite element code ABAQUS/Explicit, and a numerical methodology is developed to simulate the moving boundary problem by taking advantage of the remeshing capabilities of the code. When calibrated against centrifuge test results, the model is able to predict the resistance developed on the cone during penetration into medium and very dense sands alike, regardless of the penetration depth. Its main advantage against other published numerical methodologies employing simpler soil models such as the Drucker-Prager or the Mohr-Coulomb, is that the compressibility of the sand can be described with a single set of model parameters, irrespective of the stress level and the sand relative density.

The practical outcome of this study, resulting from a series of "numerical CPT tests", is a short comment on the estimation of parameters related to the sand density from the cone penetration resistance. Although the presented parametric analyses do not aim at replacing existing empirical relations to estimate sand properties, it is shown that the numerical methodology is able to capture the effects of sand density on large deformation problems; thus if properly calibrated to account for sand-specific parameters, it can be adapted to simulate various other moving boundary problems of practical interest.

\section{REFERENCES}

1. Susila E, Hryciw RD. Large displacement FEM modelling of the cone penetration test (CPT) in normally consolidated sand. Int. J. Numer. Anal. Meth. Geomech 2003; 27:585-602.

2. Huang W, Sheng D, Sloan SW, Yu HS. Finite element analysis of cone penetration in cohesionless soil. Computers and Geotechnics 2004; 31:517-528. 
3. Dijkstra J, Broere W, van Tol, AF. Numerical simulation of the installation of a displacement pile in sand. In: Pande, Pietruczak, editors. Numerical Models in Geomechanics NUMOG X, Taylor and Francis; 2007, p. 461-466.

4. $\mathrm{Hu}, \mathrm{S}$. Analysis of soil penetrometer using Eulerian finite element method. M.Sc. thesis, Massachusetts Institute of Technology, Cambridge, Massachusetts; 2003.

5. Yi, J. Centrifuge and numerical modelling of sand compaction pile installation. Ph.D. thesis, Department of Civil Engineering, National University of Singapore; 2009.

6. Jamiolkowski M, Ladd CC, Germaine JT, Lancellotta R. New developments in field and laboratory testing of soils. Theme lecture In: Proc. 11th Int. Conf. Soil. Mech. Found. Engng., San Francisco;1985, p. 57-156.

7. Bolton MD, Gui MW, Garnier J, Corte JF, Bagge G, Laue J, Renzi R. Centrifuge cone penetration tests in sand. Géotechnique 1999; 49:543-552.

8. DiMaggio FL, Sandler IS. Material model for granular soils. ASCE Journal of Engineering Mechanics Division 1971; 97:935-950.

9. Lade PV. Elasto-plastic stress-strain theory for cohesionless soil with curved yield surface. International Journal of Solids and Structures 1977; 19:1019-1035.

10. Vermeer PA. A double hardening model for sand. Géotechnique 1978; 28:413433.

11. Nova R, Wood MD. A constitutive model for sand in triaxial compression. Int. J. Numer. Anal. Meth. Geomech 1979; 3: 255-278.

12. Jefferies MG, Shuttle DA. Dilatancy in general Cambridge-type models. Géotechnique 2002; 52:625-638.

13. Jefferies MG, Shuttle DA. NorSand: Features, calibration and use. Soil Constitutive Models, ASCE Geotechnical Special Publication; 2005, GSP 128:204-235.

14. Dafalias YF. On cyclic and anisotropic plasticity: (i) A general model including material behavior under stress reversals, (ii) Anisotropic hardening for initially orthotropic materials. PhD Thesis, University of California, Berkeley; 1975.

15. Dafalias YF. Bounding surface plasticity. I: mathematical foundation and hypoelasticity. Journal of Engineering Mechanics ASCE 1986; 112(EM9):966987.

16. Pastor M, Zienkiewicz OC, Leung KH. Simple model for transient soil loading in earthquake analysis. II. Non-associated models for sands. Int. J. Numer. Anal. Meth. Geomech 1985; 9:477-498.

17. Whittle AJ. A constitutive model for overconsolidated clays with application to cyclic loading of friction piles. PhD Thesis, Massachusetts Institute of Technology; 1987.

18. Al-Tabbaa A, Wood DM. An experimentally based bubble model for clay. In: Pande, Pietruczak, editors. International Conference on Numerical Models in Geomechanics NUMOG III, Balkema; 1989, p. 91-99.

19. Asaoka A, Nakano M, Noda T. Superloading yield surface concept for highly structured soil behaviour. Soils Found 2000; 40(2):99-110.

20. Pestana JM, Whittle AJ. Formulation of a unified constitutive model for clays and sands. International Journal for Numerical and Analytical Methods in Geomechanics 1999; 23: 1215-1243. 
21. Abaqus- User's Manual - version 6.11. Dassault Systems Simulia Corp; 2011.

22. Bolton MD, Gui MW. The study of relative density and boundary effects for cone penetration tests in centrifuge. Report CUED/D-SOILS/TR256, Cambridge University Engineering Department, UK; 1993.

23. Been K, Jefferies MG. A state parameter for sands. Géotechnique 1985; 35(1): 99-112.

24. Jamiolkowski M, Lo Presti CDF, Manassero M. Evaluation of relative density and shear strength of sands from CPT and DMT. Soil Behavior and Soft Ground Construction, ASCE Geotechnical Special Publication; 2001, GSP 119:201-229.

25. Belotti R, Crippa V, Pedroni S, Ghionna VN. Saturation of sand specimen for calibration chamber tests. Proceedings ISOPT-1, Orlando; 1988 2:661-672.

26. Schmertmann $\mathrm{JH}$. An updated correlation between relative density $D_{R}$ and Fugro-type electric cone bearing, $\mathrm{q}_{\mathrm{c}}$. Contract Report DACW 39-76 M 6646 WES, Vicksburg, Miss.;1976.

27. Yao YP, Sun DA, Luo T. A critical state model for sands dependent on stress and density. Int. J. Numer. Anal. Meth. Geomech 2004; 28:323-337.

28. Sheng D, Sloan SW, Yu HS. Aspects of finite element implementation of critical state models. Computational Mechanics 2000; 26(2):185-196.

29. Abbo AJ, Sloan SW. An automatic load stepping algorithm with error control. International Journal for Numerical Methods in Engineering 1996; 39(10):17371759.

30. Sloan SW, Abbo AJ, Sheng D. Refined explicit integration of elastoplastic model with automatic error control. Engineering Computations 2001; 18(1-2):121-154.

31. Senders M. Cone resistance profiles for laboratory tests in sand. In: Proceedings, $2^{\text {nd }}$ International Symposium on Cone Penetration Testing CPT10, Huntington Beach, CA; 2010.

32. Loukidis D, Salgado R. Analysis of the shaft resistance of non-displacement piles in sand. Géotechnique 2008; 58(4):283-296.

33. Uesugi $\mathrm{M}$, Kishida $\mathrm{H}$, Uchikawa $\mathrm{Y}$. Friction between dry sand and concrete under monotonic and repeated loading. Soils Found 1990; 30(1):115-128.

34. Vardoulakis I, Sulem J. Bifurcation Analysis in Geomechanics. London: Blackie Academic and Professional; 1995.

35. Nemat-Nasser S, Okada N. Radiographic and microscopic observation of shear bands in granular materials. Géotechnique 2001; 51(9):753-765.

36. Mahutka KP, Konig F, Grabe J. Numerical modelling of pile jacking, driving and vibratory driving. In: Proceedings of International Conference on Numerical Modelling of Construction Processes in Geotechnical Engineering for Urban Environment; 2006, p.235-246.

37. Silva MF, White DJ, Bolton MD. An analytical study of the effect of penetration rate on piezocone tests in clay. Int. J. Numer. Anal. Meth. Geomech 2006;30(6):501-527.

38. Yi JT, Goh SH, Lee FH, Randolph MF. A numerical study of cone penetration in fine-grained soils allowing for consolidation effects. Geotechnique 2012; 62 (8):707-719.

39. Schofield AN. Cambridge geotechnical centrifuge operations. Geotechnique 1980; 30(3):227-268. 
40. Mayne PW. The second James K. Mitchell Lecture Undistrurbed sand strength from seismic cone tests. Geomechanics and Geoengineering: An International Journal 2006; 1(4):239-257.

41. Robertson PK. Interpretation of cone penetration tests - a unified approach. Can. Geotech. J. 2009; 46:1337-1355.

42. Jefferies M, Been K. Soil Liquefaction. A Critical State Approach. Taylor \& Francis; 2006.

43. Sladen JA. Problems with interpretation of sand state from cone penetration test. Geotechnique 1989; 39(2):323-332. 